\title{
Matemática Aplicada no Acoplamento Convecção-Radiação Térmica num Escoamento em Canais de Placas Planas num Meio Participante
}

\author{
DINIZ, L. S., SANTOS, C. A. C., SILVA, F. C. \\ Instituto Federal de Educação, Ciência e Tecnologia da Paraíba - IFPB Campus João Pessoa \\ Av. $1^{\circ}$ de Maio, 720, Jaguaribe, João Pessoa - PB, E-mail: lasiniz@ hotmail.com \\ Universidade Federal da Paraíba - Departamento de Engenharia Mecânica, Campus I \\ Cidade Universitária, João Pessoa, PB, E-mail: cabral@les.ufpb.edu.br \\ Instituto Federal de Educação, Ciência e Tecnologia do Ceará -IFCE Campus Crato \\ Rodovia CE 292, km 15, Gisélia Pinheiro.Crato - CE, E-mail: camilo@ifce.edu.br
}

\begin{abstract}
Resumo: No presente trabalho realiza-se um estudo onde a convecção e a radiação, estão relacionadas com a transferência de calor no escoamento em dutos de placas paralelas aplicando a Técnica da Transformada Integral Generalizada (GITT) para produzir uma solução híbrida para o modo de transferência convectiva e radiativa. Em seguida resultados de interesse prático são calculados e exibidos graficamente como a lei de espalhamento exerce no número de Nusselt local e no campo de temperatura, onde os efeitos da radiação, condução e convecção ocorrem simultaneamente e apresentam grande complexidade, tanto do ponto de vista físico como matemático, tendo despertado ao longo dos anos grande interesse dos pesquisadores, que se encontra em muitas aplicações na indústria e em outros setores da engenharia. A influência da radiação é determinada pelo método de aproximação analítica associada ao polinômio de Legendre, e analisados quanto à convergência, estabilidade e tempo computacional.
\end{abstract}

\section{INTRODUÇÃO}

Nos problemas de transferência de calor a radiação térmica é tão ou mais importante que a condução no processo interno de transferência de calor. Para a determinação precisa do campo térmico transiente é imprescindível o tratamento simultâneo destes dois processos de transferência de calor, pois, segundo Özişik (1984), a análise separada por radiação ou convecção pode introduzir erros consideráveis nos resultados obtidos. A equação da energia, de natureza não linear, sujeita a condições de contorno do primeiro tipo é resolvida empregando-se a metodologia da GITT apresentada por Cotta (1993). Na obtenção da solução foi utilizado um problema de autovalor auxiliar que permite construir uma base, a partir das autofunções, dos autovalores e das normas obtidas, que será usada para construir o potencial desejado. A parte radiativa do problema, caracterizada por uma equação não linear do tipo integro-diferencial, sujeita a condições de contorno semitransparentes que emitem e refletem difusamente a radiação térmica. Neste método, a forma integral da equação da transferência radiativa é transformada em um conjunto de equações algébricas para um meio semitransparente. Para a determinação precisa do campo térmico transiente é imprescindível o tratamento simultâneo destes dois processos de transferência de calor, pois, segundo Özişik (1984),

\section{FORMULAÇÃO DO PROBLEMA}

Considere o escoamento completamente desenvolvido de um fluido incompressível, participante, com propriedades térmicas constantes, que absorve, emite e espalha anisotropicamente a radiação térmica incidente, conforme ilustrado na Fig. (1). 


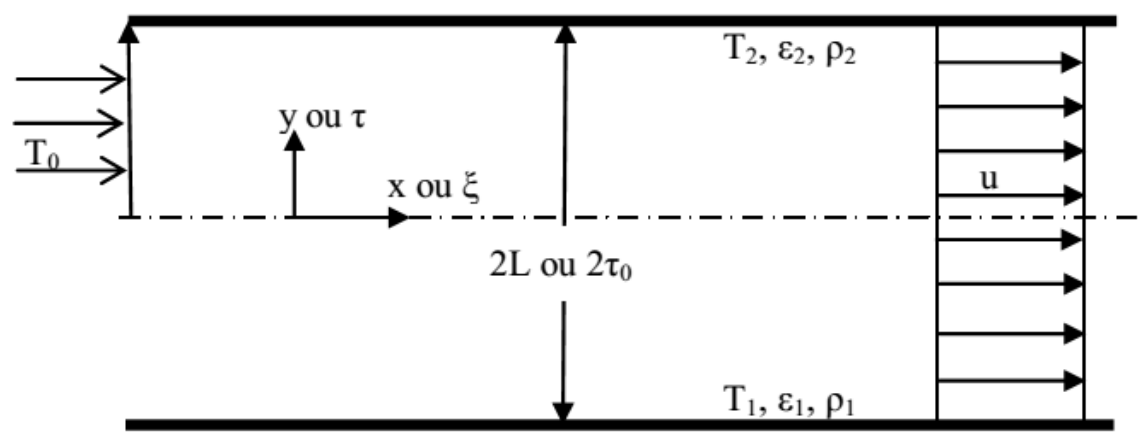

Figura 1. Escoamento entre placas planas de um fluido participante.

A equação da energia, condições de contorno e de entrada são definidas como:

Equação da energia:

$$
\rho C_{P} u_{m} \frac{\partial T(x, t)}{\partial t}=\frac{\partial}{\partial x}\left[K(T) \frac{\partial T(x, t)}{\partial x}\right]-\frac{\partial q^{r}(x ; t)}{\partial x}, \quad-L<x<L, \quad t>0
$$

Condições de contorno:

$$
\begin{gathered}
T(x, t)=T_{1}, \text { em } y=-L \\
T(x, t)=T_{2}, \text { em } y=L
\end{gathered}
$$

Condição de entrada:

$$
T(x, t)=T_{0}, \text { em } x=0
$$

onde o divergente da radiação que aparece na Eq.(1) é definido por:

$$
\frac{\partial q^{r}(x ; t)}{\partial x}=(1-\omega) \beta\left[4 n^{2} \sigma T^{4}(x, t)-2 \pi \int_{-1}^{1} P_{n}(\mu) I(x, \mu) d \mu\right]
$$

sendo que $\omega$ é o albedo para espalhamento simples, $\beta$ é o coeficiente de extinção do meio, $\mathrm{n}$ é o índice de refração, $\sigma$ é a constante de Stefan-Boltzmann e $P n(\mu)$ é a função de fase do espalhamento, expressaram a função de fase em termos de polinômios de Legendre da seguinte forma:

$$
p_{v}\left(\mu_{0}\right)=\sum_{j=0}^{\infty} a_{j} P_{j}\left(\mu_{0}\right), \quad a_{0}=1
$$

\section{ADIMENSIONALIZAÇÃO DO PROBLEMA}

Com o objetivo de se resolver uma classe de problemas que sejam definidos pelo modelo proposto serão utilizados os seguintes grupos e parâmetros adimensionais:

$$
\begin{array}{ccr}
\tau=\beta x & \xi=\frac{K \beta^{2} x}{\rho u_{m} C_{P}}=16 \tau_{0}^{2} \frac{x / D_{e}}{\operatorname{Re} \cdot \operatorname{Pr}} & \Theta(\tau, \xi)=\frac{T(x, t)}{T_{r}} \\
Q^{R}(\tau ; \xi)=\frac{q^{r}(x ; t)}{4 n^{2} \sigma T_{r}^{4}} & N=\frac{K \beta}{4 n^{2} \sigma T^{4}} & \psi(\tau, \mu)=\frac{\pi \cdot I(\tau, \mu)}{n^{2} \sigma T_{r}^{4}}
\end{array}
$$




$$
G_{n}^{*}(\tau)=\frac{G_{n}(\tau)}{4 n^{2} \sigma T_{r}^{4}}
$$

Fazendo uso dos parâmetros e grupos adimensionais definidos acima e considerando as propriedades térmicas constantes, a equação da energia (1), as condições de contorno (2) e (3), a condição de entrada (4) e o divergente da radiação vão tomar as seguintes formas adimensionais:

Equação da Energia adimensionalizada:

$$
\frac{\partial \Theta(\tau, \xi)}{\partial \xi}=\frac{\partial^{2} \Theta(\tau, \xi)}{\partial \tau^{2}}-\frac{1}{N} \frac{\partial Q^{R}(\tau ; \xi)}{\partial \tau}, \quad-\tau_{0}<\tau<\tau_{0}, \quad \xi>0
$$

Condições de contorno adimensionais:

$$
\begin{gathered}
\Theta(\tau, \xi)=\theta_{1}, \tau=-\tau_{0}, \xi>0 \\
\Theta(\tau, \xi)=\theta_{2}, \tau=\tau_{0}, \xi>0
\end{gathered}
$$

Condição de entrada adimensionalizada:

$$
\Theta(\tau, \xi)=\theta_{0}, \quad-\tau_{0} \leq \tau \leq \tau_{0}, \quad \xi=0
$$

Divergente da radiação na forma adimensional:

$$
\frac{\partial Q^{R}(\tau ; \xi)}{\partial \tau}=(1-\omega)\left\{[\Theta(\tau, \xi)]^{4}-G_{n}^{*}(\tau, \mu)\right\}
$$

onde;

$$
G_{n}^{*}(\tau)=\frac{1}{2}\left[\int_{0}^{1} P_{n}(\mu) \psi^{+}(\tau, \mu) d \mu+(-1)^{n} \int_{0}^{1} P_{n}(\mu) \psi^{-}(\tau,-\mu) d \mu\right]
$$

é a radiação incidente generalizada adimensional e $\psi(\tau,-\mu)$ satisfaz a equação da transferência radiativa definida abaixo:

$$
\begin{gathered}
\mu \frac{\partial \psi(\tau, \mu)}{\partial \tau}+\psi(\tau, \mu)=(1-\omega) \Theta^{4}(\tau, \xi)+\frac{\omega}{2} \int_{-1}^{1} p\left(\mu, \mu^{\prime}\right) \psi\left(\tau, \mu^{\prime}\right) \mathrm{d} \mu^{\prime}, \quad-\tau_{0}<\tau<\tau_{0}, \quad-1 \leq \mu \leq 1 \\
\psi^{+}\left(-\tau_{0}, \mu\right)=\varepsilon_{1} \theta_{1}^{4}+2 \rho_{1} \int_{0}^{1} \psi^{-}\left(-\tau_{0},-\mu^{\prime}\right) \mu^{\prime} \mathrm{d} \mu^{\prime}, \quad \mu>0 \\
\psi^{-}\left(\tau_{0},-\mu\right)=\varepsilon_{2} \theta_{2}^{4}+2 \rho_{2} \int_{0}^{1} \psi^{+}\left(\tau_{0}, \mu^{\prime}\right) \mu^{\prime} \mathrm{d} \mu^{\prime}, \quad \mu>0
\end{gathered}
$$

onde $\mu$ é o cosseno do ângulo formado entre o eixo positivo dos x e a direção da intensidade de radiação, $n$ é o índice de refração do meio, $\rho_{\mathrm{i}}(\mathrm{i}=1,2)$ e $\varepsilon_{\mathrm{i}}(\mathrm{i}=1,2)$ representam respectivamente as refletividades e as emissividades das superfícies de contorno.

\section{RESULTADOS E DISCUSSÕES}

A tabela (2) apresenta os resultados obtidos usando linguagem de programação FORTRAN. Fazendo uma análise nos dados pode-se perceber que o campo de temperatura converge, na quarta casa decimal, para vinte e cinco termos $(\mathrm{Nc}=25)$ de expansão no processo de transformação integral da equação da energia. Os resultados foram obtidos considerando espalhamento isotrópico da radiação e os seguintes parâmetros: $\mathrm{N}=0.5, \tau_{0}=1.0, \omega=0.5, \theta_{1}=1.0$, $\theta_{2}=1.0, \theta_{0}=0.0, \varepsilon_{1}=\varepsilon_{2}=1.0, \xi=0.0025$. 
Tabela 2. Análise de convergência do campo de temperatura adimensional.

\begin{tabular}{c|c|c|c|c|c|c}
\hline $\boldsymbol{\tau} / \mathbf{2} \tau_{\mathbf{0}}$ & $\mathbf{N c}=\mathbf{0 5}$ & $\mathbf{N c}=\mathbf{1 0}$ & $\mathbf{N c}=\mathbf{1 5}$ & $\mathbf{N c}=\mathbf{2 0}$ & $\mathbf{N c}=\mathbf{2 5}$ & $\mathbf{N c}=\mathbf{3 0}$ \\
\hline $\mathbf{- 0 . 5 0}$ & 1.0000 & 1.0000 & 1.0000 & 1.0000 & 1.0000 & 1.0000 \\
\hline $\mathbf{- 0 . 4 0}$ & 0.1574 & 0.1606 & 0.1606 & 0.1608 & 0.1609 & 0.1609 \\
\hline $\mathbf{- 0 . 3 0}$ & 0.0131 & 0.0083 & 0.0084 & 0.0084 & 0.0084 & 0.0084 \\
\hline $\mathbf{- 0 . 2 0}$ & -0.0014 & 0.0034 & 0.0034 & 0.0034 & 0.0034 & 0.0034 \\
\hline $\mathbf{- 0 . 1 0}$ & 0.0078 & 0.0031 & 0.0031 & 0.0031 & 0.0031 & 0.0031 \\
\hline $\mathbf{0 . 0 0}$ & -0.0015 & 0.0030 & 0.0030 & 0.0030 & 0.0030 & 0.0030 \\
\hline $\mathbf{0 . 1 0}$ & 0.0078 & 0.0031 & 0.0031 & 0.0031 & 0.0031 & 0.0031 \\
\hline $\mathbf{0 . 2 0}$ & -0.0014 & 0.0034 & 0.0034 & 0.0034 & 0.0034 & 0.0034 \\
\hline $\mathbf{0 . 3 0}$ & 0.0131 & 0.0083 & 0.0084 & 0.0084 & 0.0084 & 0.0084 \\
\hline $\mathbf{0 . 4 0}$ & 0.1574 & 0.1606 & 0.1606 & 0.1608 & 0.1609 & 0.1609 \\
\hline $\mathbf{0 . 5 0}$ & 1.0000 & 1.0000 & 1.0000 & 1.0000 & 1.0000 & 1.0000 \\
\hline $\begin{array}{c}\text { Tempo } \\
\text { Computacional }(\mathbf{s})\end{array}$ & $\mathbf{2 5 9 4}$ & $\mathbf{7 2 3 6}$ & $\mathbf{1 2 2 3 4}$ & $\mathbf{3 0 6 2 1}$ & $\mathbf{5 3 6 3 4}$ & $\mathbf{8 3 5 3 3}$ \\
\hline
\end{tabular}

Nas figuras (2) e (3) pode-se observar o efeito que lei de espalhamento anisotrópico exerce no número de Nusselt local e no campo de temperatura do meio. Na simulação foram adotados os seguintes parâmetros adimensionais: parâmetro condução-radiação, $\mathrm{N}=0.5$, albedo para espalhamento simples, $\omega=0.5$, as temperaturas das placas do canal, $\theta_{1}=\theta_{2}=1.0$, a temperatura de entrada, $\theta_{0}=0.0$, as emissividades e refletividades das placas dadas, respectivamente, por $\varepsilon_{1}=\varepsilon_{2}=1.0$ e $\rho_{1}=\rho_{2}=0.0$.

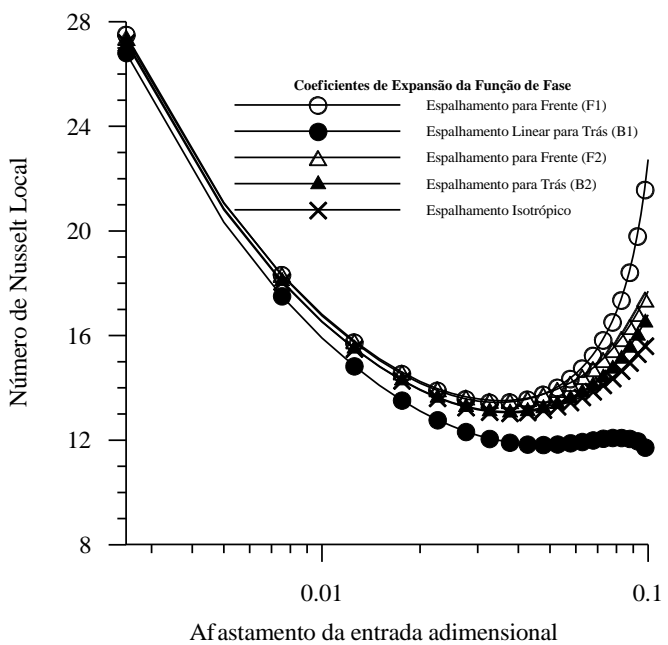

Figura 2. Efeito dos coeficientes de expansão da função de fase no número de Nusselt local.

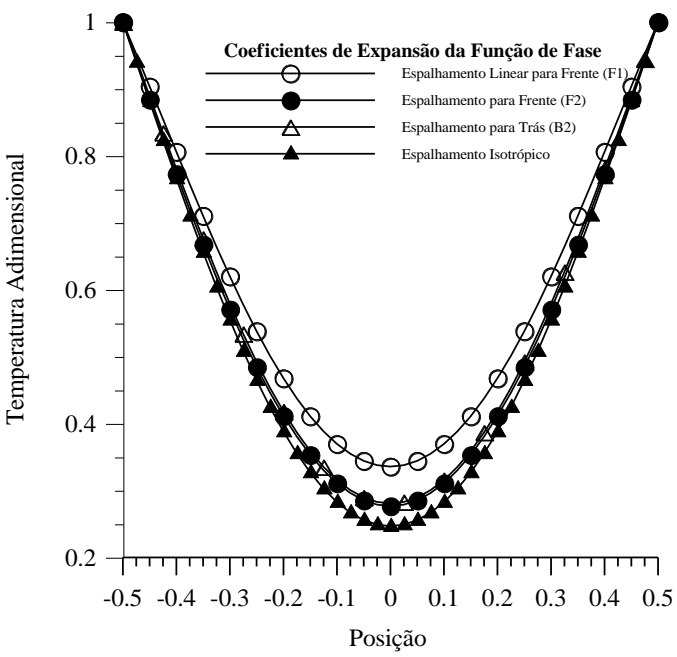

Figura 3. Efeito dos coeficientes de expansão da função de fase na distribuição de temperatura.

\section{CONCLUSÕES}

O problema estudado neste trabalho determina a transferência de calor por convecção e radiação, que ocorre em um escoamento em um canal de placas paralelas, que absorve, emite e espalha a radiação. E que para se determinar o campo de temperatura no corpo, foi necessário resolver duas equações, a equação da energia e a equação da transferência radiativa, que estão acopladas, seu tratamento analítico, sem deixar de levar em consideração os fenômenos físicos envolvidos, levando-se em consideração a contagem de sinal, como fator de acoplamento convecção-radiação, albedo para espalhamento simples, espessura óptica, número de Nusselt e dependência térmica da condutividade e difusividade, exercem na distribuição de temperatura. 


\section{REFERÊNCIAS}

[1] COTA, R., M.; "Integral Transforms in Computational Heat and Fluid Flow"; CRCPress, Boca Raton, FL, EUA, 1993.

[2] Dinz, L. S., Santos, P. H. D., Carvalho, M., Santos, C. A. C., 2003, "Theorical Analysis of The Channel Flow with Radiation in Participating Media through the use of the Generalized Integral Transform Technique", 18th International Congress of Mechanical Engineering, OuroPreto, MG.

[3] FORTRAN POWERSTATION, Version 4.0; Microsoft Corporation. 1980.

[4] Lee, H. e Buckius, R. O., 1982, "Scaling Anisotropic Scattering in Radiation Heat Transfer for a Planar Medium", Trans. ASME-Journal of Heat Transfer, Vol. 104, pp. 68-75.

[5] MIKHAILOV, M. D.; and VULCHANOV, N. L.; "Computational procedure for SturnLiouville problems", Journal of computational Physics, v. 5, 323-336, 1983.

[6] MIKHAILOV, M. D.; ÖZISIK, M. N.; "Unified Analysis and Solutions of Heat and Mass Diffusion”, John Wiley, New York, 1984.

[7] ÖSIZIK, M. N.; MURRAY, R. L.; "On the solution of linear diffusion problems with variable boundary conditions parameters”; J. Heat Transfer, V. 96, pp. 48-55, 1984.

[8] SILVA, F. C.; “Análise da Convecção e Radiação do Escoamento de Meio Participante em Dutos de Placas Paralelas Via GITT"; Dissertação de mestrado, CT/UFPB, 2013. 